\title{
El mundo narrativo en los progymnasmata de Nicéforo Basilakes
}

Recibido: 4/6/2018. Aceptado: 26/11/2018.

\section{Resumen}

Esta contribución espera demostrar que las etopeyas del rétor bizantino Nicéforo Basilakes (s. XII) exhiben una nueva narratividad, fundada en la mímesis de un mundo narrativo (storyworld) artificial y antinatural, contrafáctico y fabuloso, pero no al modo de la literatura mítica o retórica tradicional. Así inaugura un género discursivo intermedio que, contra todas las apariencias, ya no es propiamente el de los progymnasmata. Esta nueva narratividad se manifiesta a través de ciertos elementos diferenciales del mundo narrado, que incluyen una particular situación de la mujer; una nueva perspectiva sobre Eros, la sexualidad y, más en general, el amor; una religiosidad pagana que no coincide con ninguna religiosidad histórica; una espacialidad y una temporalidad no homólogas a las del mundo del autor y el lector.

\section{Storyworld in Nicephorus Basilakes' Progymnasmata}

\begin{abstract}
In this contribution I try to show that the ethopoeias of the Byzantine rhetor Nicephorus Basilakes (12th c.) feature a novel narrativity, based upon mimesis of an artificial and antinatural storyworld that is counterfactual and fabulous, though not in the way of traditional mythic or rhetorical literature. He thus propounds an intermediate discursive genre that, despite all appearances, is not properly that of progymnasmata. This new narrativity is visible through a number of differential elements of the narrated world, which include a peculiar position of the woman; a novel perspective on Eros, sexuality, and more in general on love; a pagan religiosity different from any historical religiosity; a spatiality and temporality non-homogeneous to that of the author and the reader.
\end{abstract}

Palabras clave Basilakes retórica progymnasmata Bizancio narratología

\section{Keywords}

Basilakes rhetorics progymnasmata Byzantium narratology 
1. Basilakes se ubicaría a mitad de camino entre las tendencias antiguas, que resultaban aburridas a sus contemporáneos, y ciertas corrientes modernas qu estaban pr búsqueda ecléctica de equilibrio reside también su uso del humor, que nunca es un fin en sí mismo y siempre está asociado a preocupaciones de otro orden (educar, persuadir, etc) Una concisa ia en general, así como de su situación histórica, aparece en Magdalino (2002:329)... (Continúa en página 2.)

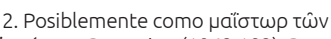
p̀ntópwv, Browning (1962:183). Para una discusión de varias posibilidades, cfr. Garzya (1973:5, n.20).

3. Es decir, maestro acerca de las epístolas paulinas; cfr. Garzya (1969:66, n. 39). Browning (1962:170-171), refiriéndose a otro maestro de la escuela patriarcal de Constantinopla, Miguel ò toû $\Theta \varepsilon \sigma \sigma a \lambda o v i ́ k n \zeta$, refiere que fue sucesivamente " $\delta ı \delta ́ \sigma k a \lambda$ os

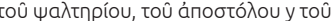

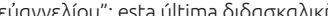
ákía era jerárquicamente superior; la seguía en importancia la de $\delta ı \delta a ́ \sigma k a \lambda o s$

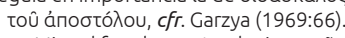
Miguel fue depuesto el mismo año que Basilakes (Browning, 1962:182).

4. "'Pro-gymnasmata' means 'preliminary exercises', preliminary that is to the practice of declamation in the schools of rhetoric, which boys usually began between the age of twelve and fifteen. The progymnasmata were assigned by Greek grammarians to students after they had learned to read and write as preparation for declamation and were continued in rhetorical schools wer writen exercises even after declamation had begun", Kennedy (2003:x). El número de progymnasmata no es idéntico en todos los tratados: Teón conoce diez, Hermógenes doce, Aftonio catorce. Entre los principales se encuentra la fábula, la narración, la etopeya o personificación, el encomio y la écfrasis. 5. Abundante bibliografía en Papaioannou (2007:357).

6. El autor no explica por qué estos textos son 'ficcionales'.

7. Esta contribución es parte de un proyecto más vasto, que por motivos de espacio no puede presentarse como un único artículo. Quedan provisoriamente único artículo. Quedan provisoriamente ciertas cuestiones atinentes al narrador (por ejemplo, su uso de los deícticos y demás aspectos formales de su técnica)

y un desarrollo más extenso de las peculiaridades de la temporalidad y espacialidad en Basilakes. Las comparaciones con los novelistas bizantinos, especialmente Eumacio Makrembolites, también están reducidas a un mínimo; para una apta reseña de lo que tienen vecho Nilsson (2014), con bibliografía.

8. La narratología suele distinguir explícitamente entre storyworldy narrative world, pero en lo que sigue utilizo los términos indistintamente para referirme al mundo evocado explícita o implícitamente en una narración.

9. Todas las citas son de la edición de Pignani (1983), cotejadas con la de Beneker; Gibson (2016).

\section{Vida y valoración moderna}

Nicéforo Basilakes, nacido en torno a 1115, inició su carrera en el ámbito de

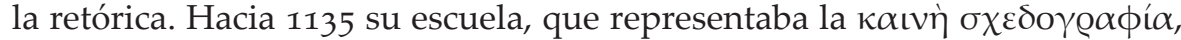
habría tenido un particular éxito (Garzya, 1973:5). ${ }^{1}$ Alrededor de 1140 obtuvo un puesto en la escuela patriarcal. ${ }^{2}$ Luego pasó a la enseñanza teológica. Se

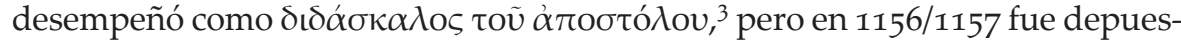
to: "Il Basilake fu [...] colpito d'anatema. Questo gli comportò la perdita del posto e l'esilio, attestati entrambi in maniera sensibile e amara dalle lettere e da coperte allusioni in altri scritti" (Garzya, 1970:316). Murió en torno a 1180 (Garzya, 1971:301-302).

Sus progymnasmata pertenecen a la primera etapa de su carrera. ${ }^{4}$ Pueden fecharse alrededor del año 1140. ${ }^{5}$ En esa década,

Eros is [...] a prominent theme and romantic fiction reappears, fusing erotic prose with poetry [...]; his [Basilakes'] erotic progymnasmata are the first Byzantine erotic fictional texts since late antiquity [...] (Papaioannou, 2007:358). ${ }^{6}$

Antes del s. XX, Basilakes era considerado un orador puro y simple. Al gran Krumbacher le despertaban interés sus sátiras en prosa al modo de Luciano, como también las practicaba Teodoro Pródromo; pero estas sátiras, se sabe, no se han conservado (Krumbacher, 1897:473-474). Le debemos a Antonio Garzya el primer acercamiento al potencial novelesco, erótico y humorístico de los progymnasmata, así como la popularidad moderna de Basilakes.

Uno de los motivos de esta popularidad se encuentra en su perspectiva sobre el amor: burlón, irónico, antinatural. El amor y la figura de Eros ya han sido estudiados como temas centrales en Basilakes (véase, por ejemplo, Magdalino, 1992; Papaioannou, 2007). En esta contribución, la representación del amor va a interesarme no tanto como tema ${ }^{7}$ sino por su función formal como elemento extrañador (instaurador de contrafacticidad). Si bien es un elemento entre varios, lo considero central para la generación del mundo narrativo, junto con otro del que es correlativo: la representación de la mujer.

\section{Storyworld y extrañamiento: mujeres $^{8}$}

Con la excepción de la virgen María, la mujer nunca es protagonista en los progymnasmata de tema bíblico. No solo no es protagonista: también está mal connotada. En una fábula ( $\mu \tilde{v} \theta o \varsigma)$ protagonizada precisamente por una dama, Basilakes se siente obligado a explicar que pone en escena a una mujer sabia porque los antiguos solían hacerlo, sugiriendo que no por eso debe ofenderse el respetable público (masculino):

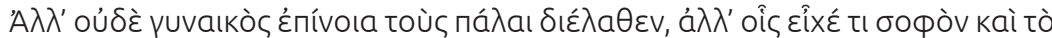

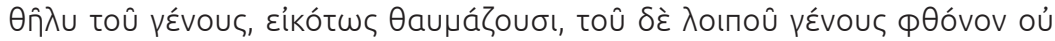
noııûvtaı toû Өaúnatoc. (Prog. 11). ${ }^{9}$

Pero no se les ocultó a los antiguos la astucia de la mujer, sino que admiran verosímilmente las cosas en las que tuvo algo sabio incluso lo femenino del género <humano>, sin incurrir por esa admiración en reproche ( $\phi \theta$ óvov) del resto $<$ masculino $>$ del género. 
La aparición de una mujer astuta debe ser disculpada explícitamente. Probablemente se trate de la única mujer de veras inteligente en todo el corpus de Basilakes - excluyendo las mujeres del mundo pagano, en el que no se verifica esta misoginia ascética. Para Basilakes, el ser real de la mujer (no solo su rol social) muta radicalmente según el entorno en el que se halla. Su visión acerca de los géneros es más constructivista que naturalista. ${ }^{10}$

En el storyworld mítico-clásico, el cambio de religiosidad y de perspectiva sobre lo real permite el surgimiento de una suerte de realidad alternativa, pagana y politeísta, situada no tanto en el pasado como en otro mundo.

Las heroínas paganas tienen un deseo manifiesto que se convierte en el tema mismo de la composición y pese a esto - o por esto mismo - las composiciones las dejan relativamente bien paradas. Una explicación acerca de la rareza de una mujer astuta sería innecesaria aquí. El storyworld mismo se encarga de dar a la mujer el contexto que la explica; ninguna necesidad de denigrarla explícitamente.

Es claro que, de todos modos, falta una visión positiva sobre lo femenino. Incluso en las etopeyas de tema pagano quienes sufren preponderantemente el amor, en particular aquel que se opone a la $\phi v ́ \sigma \iota s$, son las mujeres. Basta recordar a Mirra y a Pasifae, representadas en sendas narraciones y etopeyas. ${ }^{11}$ Ningún ser masculino recibe un tratamiento comparable. ${ }^{12}$

A diferencia de lo que sucede en el storyworld bíblico o cristiano, aquí aparecen mujeres fuertes y autónomas, con un deseo sexual bien definido y, asociadas a ellas, el amor como fuerza cósmica.

\section{Excursus: Breve historia del amor}

Para visualizar con más claridad el rol del amor en Basilakes, conviene recordar algunas de las etapas de la evolución del amor, seleccionadas de modo algo impresionista. En la Antigüedad Clásica, la apreciación era pesimista: “tanto griegos como romanos tenían una concepción negativa (o, al menos ambivalente) sobre el amor. De ahí que lo compararan literariamente con las dos actividades que se reputaban como más peligrosas en la época: la guerra (es el conocido tópico de la militia amoris) y la navegación (navigium amoris)" (Laguna Mariscal et al., 2017:126). ${ }^{13}$ Esta visión negativa sobre el amor no se extendía necesariamente a la sexualidad.

$\mathrm{Al}$ inicio de la era cristiana eran influyentes las recomendaciones paulinas según las cuales el celibato es preferible, pero el matrimonio aceptable. Con el tiempo se popularizan visiones más radicales, que coronan a la virginidad como la más elevada de las virtudes (cfr. Beck, 1986:25-34). En el mundo pagano, por su parte, las novelas griegas ponen en escena un amor heterosexual, fuertemente material y no solo espiritual, virtuoso pero no casto.

En una clara vertiente ascética, obras que en cierta medida coinciden con la perspectiva sobre el amor de la novela griega excluyen, en cambio, la concreción de la sexualidad: así, en un acta apócrifa (s. II), Tecla enloquece por la persona particular de Pablo; se enamora de su voz, solo quiere estar con él, viaja para volver a encontrarlo, etc. Se trata de un amor muy material, sin duda, pero excluye explícitamente el elemento sexual, aunque este permanezca como corriente subterránea.
10. Herman (s/a) observa en una entrevista que la narratología posclásica, en oposición a la clásica, se nutre de diversas corrientes: "Gender theory, for example, raises questions about gender identities that bear directly on models of narration and characterization in narrative". Creo que esta observación es apta para explicar la función que el rol de la mujer ejerce en el entramado de un storyworld distinto de los habitualmente disponibles para el narrador bizantino y en el surgimiento de una mímesis alternativa.

11. Prog. $19 \Delta$ Ińynua tò katà tìv

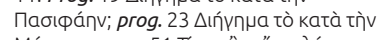

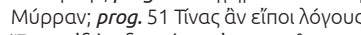

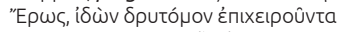

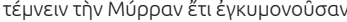

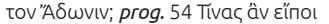

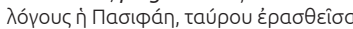
12. Sí se enamoran, aunque de modo relativamente trivial, un dios como Zeus o un héroe como Heracles.
13. Auerbach $(1958: 164$, n.28) resalta que en la Antigüedad el amor nunca o rara vez se trataba en estilo sublime (en esto consiste la gran diferencia con el estilo sublime vernáculo de la Baja Edad Media), y lo que a nosotros nos parecen excepciones, como el canto IV de la Eneida, no eran consideradas tales por un téorico como Servio (ad Aen. IV, 1): "paene comicus stilus est; nec mirum, ubi de amore tractatur". 
14. Eisagoge 40, 44-67. Sobre la plausibilidad de que el autor sea efectivamente Focio, véase Signes $\mathrm{Co}$ doñer; Andrés Santos (2007:147-160); Magdalino (2011:152), tiene ciertas dudas pero favorece la atribución: (sobre la Eisagoge) "law code drafted, probably by Photios, between 880 and 886", y nuevamente (2011:155): Focio, si no el autor, probablemente fuera "the brains behind the Eisagoge".
15. Esto muestra hasta qué punto los dos elementos están ligados. También es clave el género del público, que en el caso de los progymnasmata era mayoritariamente masculino. También su relación dialéctica con la religiosidad, para lo cual véanse las notas siguientes sobre el amor-ná oc.
16. Auerbach (1958), especialmente el apartado Gloria passionis (1958:54-63), que consiste en una reescritura de Auerbach (1941).
Posteriormente, diversas composiciones pregonan y promueven la virginidad pura; el acto sexual, incluso en el matrimonio, es vituperado; un ejemplo del s. VII, la misma época en que se difunden tendencias rigoristas contra el sexo extramatrimonial, es Leoncio de Neápolis (cfr. Fernández, 2016:51-52). En el s. IX Focio, que proponía penas tan terribles para los delitos sexuales, ${ }^{14}$ ya puede admitir que lee las novelas griegas, aunque condene su inmoralidad y diga que solo son útiles por su estilo (Lemerle, 1971:194).

Con el tiempo la educación se vuelve más secular. La homogeneización religiosa hace que el amor carnal, a menudo asociado al paganismo, deje de ser percibido como una amenaza. El matrimonio empieza a ser valorado. Kazhdan sitúa esta visión positiva sobre el matrimonio en torno al s. X (cfr. Kazhdan, 1990:132). Según Angelikí Laiou, la Iglesia quería controlar el deseo en el s. IV; con el tiempo, intentó controlar la institución matrimonial. Este esfuerzo de control se habría iniciado en el s. X y florecido en el XII (Laiou, 1992:88). La crítica efectiva de las prácticas sexuales, incluso las consideradas aberrantes, en cambio, se vuelve menos preponderante. El amor, matrimonial y de otros tipos, sube en la estima social.

El cambio ya está avanzado en el siglo de Basilakes, el XII. Con él, en particular, se asocia el renacimiento de la narración erótica y a la figura de Eros, que reaparecerá entre los novelistas bizantinos del s. XII.

\section{Storyworld y extrañamiento: amor}

Hemos visto que en Basilakes la mujer activa está confinada a los progymnasmata de tema pagano, y es un elemento central para generar el storyworld mítico-clásico. Lo mismo sucede con el amor. ${ }^{15}$

En la única etopeya basada en un texto hagiográfico ('histórico'), Eros aparece, sí, pero únicamente para explicar la concupiscencia criminal de un soldado godo (prog. 56); la novedad consiste en que no se atribuye la tentación a un demonio. En los progymnasmata de tema bíblico, el amor aparece casi exclusivamente como fuente de males. El foco no recae en el amor en sí, sino en sus consecuencias catastróficas; Eros o el amor es prácticamente sustituible por el Diablo. En las etopeyas de tema pagano, en cambio, no interesan las consecuencias del amor, sino su realidad actual y el efecto inmediato que causa en las personas que lo padecen. Es cierto que el amor no tiene final feliz, al contrario de lo que sucede en la novela. Tampoco se trata de un amor que pueda terminar en matrimonio. Al contrario, aparece el abanico casi completo de relaciones prohibidas: adulterio, fornicación, homosexualidad, zoofilia, violación, incesto entre padre e hija impulsado activamente por esta última. Sin embargo, a diferencia de lo que ocurre con las etopeyas bíblicas, la perspectiva sobre el amor es distanciada e irónica pero no crítica o negativa, salvo muy indirectamente; no se emite condena contra el sujeto enamorado ni este tiene conciencia de culpa.

El amor que pone en escena Basilakes no tiene los elementos distintivos que sí adquirirá casi contemporáneamente en Occidente con los trovadores y Chrétien de Troyes. El amor asociado a la pasión, estudiado magistralmente por Auerbach, ${ }^{16}$ tiene elementos de la passio divina: un aspecto pasivo (por su carácter de golpe sobre el alma) combinado con otro activo (por los movimientos que impulsa), sufrimiento y a la vez exaltación, felicidad y a la vez tortura, 
etc.: "Alles christliche Denken, insbesondere alle mystischen Vorstellungen bewegen sich in der Polarität des Gegensätzlichen. Auch die Gottesliebe ist Liebesqual, auch wenn sie erhört ist; denn Gott ist zu stark für die Seele" (Auerbach, 1958:61). Estas contradicciones encuentran su expresión más económicas en las paradojas antitéticas típicas de la poesía amorosa europea a partir de los provenzales, como "Pace non trovo, e non ho da far guerra" (Auerbach, 1958:60, n. 11). Auerbach observa una evolución: "die Passionmystik mit ihrer Annäherung von Passio und Ekstase [ist] auch auf die Entwicklung von passio-Leidenschaft von Einfluß gewesen" (Auerbach, 1958:62). La divinización tácita del erotismo tuvo una importancia considerable en la percepción moderna del amor. El amor de Basilakes (y, podemos agregar, el de los novelistas bizantinos) ocupa una posición intermedia entre el antiguo y el de la primera Edad Media, por un lado, y el moderno, por el otro: no se trata todavía

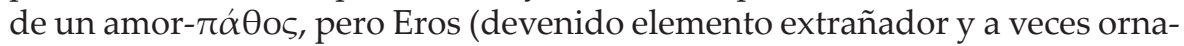
mento) ya no tiene una función cultual ni es objeto de una pietas sentida.

Como en el amor antiguo, en Basilakes no hay simetría entre las partes: el amor es unilateral y en ocasiones el $\dot{\varepsilon} \varrho \omega \mu \varepsilon v o \varsigma$ ni siquiera puede saberse objeto de deseo. ${ }^{17}$ La novela griega era más democrática en este punto; los enamorados contaban por partes iguales. En el amor europeo del siglo XII la asimetría vuelve a ganar la partida, pero en una forma diversa de la antigua y de la de Basilakes: ya no tanto por roles (activo / pasivo) como por rango y jerarquía. El objeto amado deviene una señoría (entre los provenzales) o es divinizado (stil nuovo). El primer punto (considerar al amado una suerte de señor feudal) sí aparece en Eumacio Makrembolites. ${ }^{18}$ Pero cualquiera de estas formas novedosas del amor está ausente de la obra de Basilakes.

Dante podía escribir sin ruborizarse ni rebajar su estilo "che quando / Amor mi spira, noto" ${ }^{19}$ Este rol elevado y a la vez cercano del amor, que se entrevé en Chrétien o los poetas provenzales, brilla por su ausencia tanto en un autor clásico como en Basilakes o en los novelistas bizantinos.

Basilakes presenta al amor sin distancia, pero no de un modo cercano. Sin crítica, pero no en serio. Esto se debe a que en todos los casos el corte con el mundo cotidiano del rétor y de su auditorio es completo. El amor sexual y las mujeres autónomas solo aparecen en ese storyworld mítico-clásico que, nada curiosamente, tiene muchos puntos de contactos con el de las novelas bizantinas del siglo XII.

La perspectiva ladina de Basilakes para representar el amor se funda en su posicionamiento respecto del texto, más que en las opiniones sustanciales y explícitas que brinda sin cesar. No es el posicionamiento de un novelista antiguo o medieval; tampoco el de un historiador o un poeta, y ni siquiera el de un rhétor, como intento demostrar en la próxima sección.

\section{Otro mundo}

En Basilakes, ni la historia ni la geografía son reconocibles, y sin embargo su mundo tiene poco en común con el del cuento de hadas (por su moralidad, su crueldad, su sexualidad, su relación con la escritura, el público al que apunta, etc.). Sus narraciones y etopeyas se desarrollan en una realidad alternativa habilitada por su nueva narratividad. Su tipo de mímesis, correlativa al peculiar encuadramiento del autor y del narrador, podría caracterizarse como un
17. El caso más extremo es, desde luego, el de Pasifae. Esta desproporción entre las partes, que excede la simple asimetría, es explotada por Basilakes para lograr un efecto cómico. Sobre el rol compositivo de estas incongruencias, cfr. infra.

18. Magdalino (1992:198), sobre los topoi del amor cortés que aparecen tanto en la literatura francesa como en la novela de Makrembolites.

19. Purgatorio 24, 52-53: cuando Amor lo inspira, el poeta toma nota (o registra). Para la cita y la comparación con los clásicos, Auerbach (1958:168). 


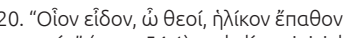

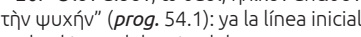
da el tono del resto del progymnasma (que podría clasificarse de sublime vulgar, como frases del estilo "romper el corazón": apuntan a un contenido elevado y tienen una formulación trillada). La frase de Servio, cit. supra, n. 17 ("paene comicus stilus est; nec mirum, ubi de amore tractatur"), que nos parece casi absurda referida a Eneida IV, puede ser aplicada literalmente a esta etopeya. De todos modos, si aquí fuera Medea quien hablara la frase podría parecer torpe, pero no cómica; inversamente, si en vez de Medea Pasifae dijera observando al toro: "Tint $\varepsilon \mu \varepsilon \delta \varepsilon i \lambda a i n v$

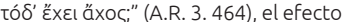
sería grotesco (de descolocación).

21. Pace Kennedy (1983:56), que considera a las Heroides una suerte de etopeyas en verso (sin detenerse en las diferencias de tratamiento). realismo simplificado en el que las figuras se esencializan, los lugares se vuelven tópicos, las acciones básicas, las psicologías sin dobles fondos: un modelo literario potencial y contrafáctico. Al mismo tiempo, el mundo narrativo está lo suficientemente circunstanciado como para diferenciarse radicalmente de cualquier mundo cotidiano o histórico. Hago una salvedad: el storyworld mítico de la tragedia o la épica clásicas tampoco coincide con un mundo cotidiano; esta es, precisamente, una de sus diferencias con la novela griega. Sin embargo, una serie de parámetros objetivos como la religiosidad, las motivaciones, el rol de la mujer y las posibilidades del amor, la causalidad suficiente de las acciones, la geografía, la relación con una historia externa posible, sí vuelven (comparado con este storyworld mítico-clásico) todavía más distante y menos naturalista - decididamente artificial, en una palabra - el mundo de los progymnasmata de Basilakes.

Por lo demás, incluso los cuentos de hadas, la épica o la tragedia producen una suerte de reconocimiento, una intuición de pacto que permite al lector $\mathrm{u}$ oyente habitarlos por el tiempo del relato. Esto, en general, sucede con la mayor parte de los mundos narrados: "Story openings prompt interpreters to take up residence (more or less comfortably) in the world being evoked by a given narrative" (Herman, 2009:79). El mundo de Basilakes es justo lo contrario. En esto se parece al mundo de los progymnasmata tardoantiguos. En particular, no favorece la identificación ni la empatía con el protagonista. No hay inmersión por parte del auditorio o involucramiento experiencial. El distanciamiento de Basilakes parece el típico de un rhétor.

Sin embargo, aplicado a temas y tratamientos novelescos, este posicionamiento que favorece el extrañamiento e impide la identificación no es inocente. Basilakes desarrolla un discurso novelesco en exceso de cualquier necesidad retórica. El puro y simple distanciamiento retórico se torna distancia irónica y extrañamiento. Así, por ejemplo, el tono del personaje se opone a la implicancia de la escena, y su autopercepción es incompatible con la percepción del auditorio; las palabras de Pasifae podrían haber sido sublimes e inspirar conmiseración en otro ámbito, pero en su situación se vuelven grotescas; ${ }^{20} \mathrm{el}$ personaje habla con máxima seriedad y esta seriedad, por el contexto, mueve a risa.

Basilakes narra impersonalmente un mundo antinaturalista (como si fuera un orador), en el que se desarrollan historias a las que da un desarrollo colorido y sensual, deteniéndose en mujeres fuertes con deseos singulares (como si fuera un novelista). Por eso sus progymnasmata, que desde algún punto de vista se parecen a los de Libanio (extrañamiento, artificialidad), desde otro están mucho más cerca de las Heroides de Ovidio (por su carácter más circunstanciado, su morosidad en estados interiores, su extensión) - mientras que los progymnasmata de Libanio y las Heroides de Ovidio, a primera vista, tienen poco en común. ${ }^{21}$

En esta hibridez, que reaparecerá en la novela bizantina de Eumacio Makrembolites, consiste una de las originalidades de Basilakes. Este invita al lector a no acomodarse, a no tomarse nada excesivamente en serio, a percibir la ironía y la distancia. La falta de identificación no concierne solo el narrador, sino también el mundo narrado (irreconocible e inhabitable). No hay inmersión del lector (reader immersion). Más en general los desplazamiento o cambios deícticos (deictic shifts), que habitualmente facilitan el involucramiento personal y funcionan como bisagra entre la experiencia narrada en el texto y la del 
lector, le sirven a Basilakes, por el contrario, para subrayar la irrealidad de su mundo narrativo. El compromiso narrativo (narrative engagement) es mediato.

Hay una ausencia del factor de empatía de la historia experiencial, característica del discurso narrativo (Fludernik, 1996:82: "empathy factor of the experiential story"). En los rétores clásicos este no puede existir, por la función y compacidad de las producciones, que exigen desplazar a la figura autoral fuera de los progymnasmata. En Basilakes es rechazado. Aquí aparece uno de los puntos centrales en los que Basilakes anticipa el posicionamiento de la figura autoral en los novelistas bizantinos, que en muchos puntos se acercan más a la de Basilakes que a la de sus 'precursores' griegos. Más que a una narración natural, Basilakes apunta a una narración antinatural, desde un ángulo que no habían practicado del todo ni los rétores ni los novelistas.

\section{Conclusión}

Los progymnasmata de Basilakes son un laboratorio narrativo que puede haber facilitado el surgimiento y el desarrollo de la narratividad novelesca del siglo XII; no es casual que cuenten con un componente narrativo diferencial frente a sus antecedentes, los progymnasmata tardoantiguos. Composicionalmente, funcionan como una pre-novela formal: ${ }^{22}$ son una colección de formas breves análogas a las que constituyen las unidades narrativas mínimas (buildingblocks) $)^{23}$ de las novelas griega y bizantina. En estas formas breves hay una tensión entre retórica y narración novelesca, entre improvisación sobre la base de formas retóricas fijas y el ejercicio efectivamente escrito, entre unidades breves y conjuntos extensos (aunque en Basilakes carezcan de ilación sintáctica). ${ }^{24}$ La narratividad de Basilakes es análoga a la de la novela en muchos puntos: 1 . el posicionamiento del autor y el narrador $y$, asociado a él, una mímesis en la que predomina el distanciamiento, la ironía y la imposibilidad de identificación; 2. un storyworld mítico-clásico contrapuesto al mundo del aquí y el ahora, con un tiempo-espacio antinaturalista y con una particular función del amor, de la mujer movida por el deseo y de la religiosidad (pagana pero no histórica).

Las etopeyas de Basilakes ya no son del todo progymnasmata, pero todavía no son novelas. Dicho con más precisión: la narratividad de muchas de sus etopeyas ya no es la de los progymnasmata tradicionales, nunca fue la de la novela griega y aun no es la de la novela bizantina, aunque apunte a ella. ${ }^{25}$ Ese carácter híbrido e impuro, de exploración y eclosión, es una de las causas del atractivo de estas etopeyas.

\section{Notas}

1 Basilakes se ubicaría a mitad de camino entre las tendencias antiguas, que resultaban aburridas a sus contemporáneos, y ciertas corrientes modernas que estaban privadas de contenido. En esta búsqueda ecléctica de equilibrio reside también su uso del humor, que nunca es un fin en sí mismo y siempre está asociado a preocupaciones de otro orden (educar, persuadir, etc.). Una concisa descripción de la schedographia
22. Ya Rohde (1914:370) los consideraba antecedentes de la novela: "Man übte sich endlich auch in der selbständigen Ausbildung erotischer Erzählungen. Wir besitzen unter den rhetorischen Progymnasmen eine zierlichen Erzählung alter erotischer Legenden. Da begegnen uns die alten wohlbekannten Abenteuer des Achill und der Penthesilea, Pyramus und Thisbe, Atalante und Hippomenes usw." 23. Es el término que O'Sullivan (1994:30) propone en su estudio sobre Jenofonte de Éfeso: "The Ephesiaca is to a large extent a complex of fairly narrow repertoire of recurrent simple happenings and concepts. They are used as building-blocks in the story, sometimes singly, sometimes in varying combinations, often in more or less standard and extensive combinations that go to make up those scenes, or themes, that recur in the romance."

24. También en Makrembolites hay una unidad lírica o espacial, más que sucesiva o temporal; ver en último término Fernández (2018).

25. No pretendo participar de la animada discusión acerca de la cronología relativa de Basilakes y Makrembolites. Makrembolites podría ser anterior en el tiempo; esto no impide que desde el punto de vista de desarrollo de una forma Basilakes sea precursor, si no históricamente sí en el sentido borgeano de "Kafka y sus precursores" en Borges (1952). 
en general, así como de su situación histórica, aparece en Magdalino (2002:329): "[...] the eleventh century saw two developments in institutional schooling which almost certainly increased the availability of education in Constantinople. One was the introduction of a school exercise, schedographia, which substituted the intensive study of short, dictated, model compositions for the extensive study of entire ancient texts as a means of learning grammar, syntax, etymology and orthography. [...] It was suited not only to large classes, but also to pupils who were not well supplied with time, books and writing materials. It offered a short cut to the acquisition of fairly advanced literate skills [...]." (Viene de página 2.) 


\section{Q Bibliografía}

" Auerbach, E. (1941). "Passio als Leidenschaft", Publications of the Modern Language Association of America 56, 1176-1196.

" Auerbach, E. (1958). Literatursprache und Publikum in der lateinischen Spätantike und im Mittelalter. Bern: Francke.

» Beck, H.-G. (1986). Byzantinisches Erotikon. München: Beck.

» Beneker, J.; Gibson, C.A. (2016). The Rhetorical Exercises of Nikephoros Basilakes. Progymnasmata from Twelfth-Century Byzantium. Cambridge -London: Harvard University Press.

" Borges, J.L. (1952). Otras inquisiciones. Buenos Aires: Sur.

" Browning, R. (1962). "The Patriarchal School at Constantinople in the Twelfth Century", Byzantion 32, 167-202.

"Fernández, T. (2016). "Malas mujeres en Leoncio de Neápolis", Rivista di Studi Bizantini e Neoellenici 53, 43-58.

" Fernández, T. (2018) "La temporalidad en la novela griega y bizantina", Stylos (en prensa).

" Fludernik, M. (1992). Towards a "Natural"Narratology. London-New York: Routledge.

» Garzya, A. (1969). "Intorno al Prologo di Niceforo Basilace", Jahrbuch der Österreichischen Byzantinistik 18, 57-73.

» Garzya, A. (1970). "Precisazione sul processo di Niceforo Basilace", Byzantion 40, 309316.

" Garzya, A. (1971). "Fin quando visse Niceforo Basilace?", Byzantinische Zeitschrift 64, 301-302.

» Garzya, A. (1973). "Literarische und rhetorische Polemiken der Komnenenzeit", Byzantinoslavica 34, 1-14.

» Herman, D. (2009). "Narrative Ways of Worldmaking". En: Heinen, S.; Sommer, R. (eds.), Narratology in the Age of Cross-Disciplinary Narrative Research. Berlin-New York: De Gruyter, 71-87.

"Herman, D. (s/a). "New Developments in the Study of Narrative: An Interview with David Herman". En: http://cf.hum.uva.n//narratology/a11_an_interview_with_david_ herman.htm.

" Kazhdan, A. (1990). "Hagiography and Sex in the Fifth to Twelfth Centuries", DOP44, 131-143.

» Kennedy, G. A. (1983). Greek Rhetoric under Christian Emperors. Princeton: Princeton University Press.

» Kennedy, G. A. (2003). Progymnasmata. Greek Textbooks of Prose Composition and Rhetoric. Atlanta: Society of Biblical Literature.

» Krumbacher, K. (1897). Geschichte der byzantinischen Litteratur. München: Beck.

»Laguna Mariscal, G.; Gómez Luque, J.A.; Martínez Ariego, M. (2017). "'Entre golfos anda el juego': el tópico literario del navigium amoris en la poesía goliárdica", Minerva $30,123-152$ 
" Laiou, A.E. (1992). Mariage, amour et parenté à Byzance aux XIe-XIII siècles. Paris: De Boccard.

» Lemerle, P. (1971). Le premier humanisme byzantin. Paris: PUF.

" Magdalino, P. (1992). "Eros the King and the King of Amours. Some Observations on Hysmine and Hysminias", DOP 46, 197-204.

" Magdalino, P. (2002) [1993]. The Empire of Manuel I Komnenos, 1143-1180. Cambridge: Cambridge University Press.

» Magdalino, P. (2011). "Orthodoxy and History in Tenth-Century Byzantine 'Encyclopedism'". En Van Deun, P.; Macé, C. (eds.), Encyclopedic Trends in Byzantium? Proceedings of the International Conference held in Leuven, 6-8 May 2009. LeuvenParis-Walpole: Peeters.

» Nilsson, I. (2014). Raconter Byzance: la littérature au XIle siècle. Paris: Les Belles Lettres.

» O'Sullivan, J.N. (1994). Xenophon of Ephesus. Berlin-New York: De Gruyter.

»Papaioannou, S. (2007). "On the stage of Eros: Two Rhetorical Exercises by Nikephoros Basilakes". En: Grünbart M. (ed.), Theatron. Rhetorische Kultur in Spätantike und Mittelalter. Berlin-New York: De Gruyter, 357-376.

" Pignani, A. (1983). Niceforo Basilace. Progimnasme e monodie. Testo critico, introduzione, traduzione. Napoli: Bibliopolis.

» Rohde, E. (1914). Der griechische Roman und seine Vorläufer. Leipzig: Druck.

» Signes Codoñer, J.; Andrés Santos, F.J. (2007). La Introducción al derecho (Eisagoge) del patriarca Focio, Madrid: CSIC. 